\title{
ОБ ОДНОМ ВОПРОСЕ ИЗ КОУРОВСКОЙ ТЕТРАДИ
}

\section{В. А. Середа, А. И. Созутов}

\section{В 11-м издании Коуровской тетради [1] А. В. Тимофеенко поставил следующий}

Вопрос 11.101. Существует ли группа Голода (см. 9.76) с конечным центром?

В литературе пока нет единого мнения, какие группы называть группами Голода. Так в вопросе 8.67 [1] под группой Голода понимается конечно порожденная ненильпотентная подгруппа присоединенной группы ассоциативного нилькольца. В вопросе А. В. Тимофеенко, как и в 9.76 [1], речь идет о более узком классе групп. Для ясности приведем вопрос В. П. Шункова 9.76 из Коуровской тетради [1].

Вопрос 9.76. Назовем группой Голода $r$-порожденную, $r \geqslant 2$, подгруппу гр $\left(1+x_{1}+I\right.$, $\left.1+x_{2}+I, \ldots, 1+x_{r}+I\right)$ присоединенной группь $1+F / I$ фактор-алгебры $F / I$, где $F-$ свободная алгебра многочленов без свободного члена от неперестановочных переменных $x_{1}, x_{2}, \ldots, x_{r}$ над полем характеристики $p>0$, a $I$ - такой ее идеал, что $F / I$ - ненильпотентная нильалгебра (см. Е.С. Голод, Изв. АН СССР, 1964, сер. матем., 28, № 1 , c. 273-276). Доказать, что в группах Голода иентрализатор любого элемента бесконечен.

Заметим, что Тимофеенко (Матем. заметки, 39, № 5 (1986), с. 647-650) построил группы Голода с бесконечным иентром; независимо и другим способом такой же результат получил в середине 90-х г. Л. Гамуди (L. Haтmоudi).

В данной заметке под группой Голода понимается группа Голода в смысле Шункова. Положительный ответ на вопрос 11.101 Тимофеенко вытекает из следующей теоремы.

Теорема. Если P - группа Голода, то некоторая ее фактор-группа является группой Голода и имеет тривиалъный центр.

Итак, пусть $F=\Phi\left(x_{1}, \ldots, x_{d}\right)$ - ассоциативная алгебра многочленов над полем $\Phi$ простой характеристики $p$ от неперестановочных переменных $x_{1}, \ldots, x_{d}$. Алгебра $F$ градуирована, т.е. разлагается в прямую сумму $F=F_{0} \oplus F_{1} \oplus \cdots$ подпространств $F_{n}$, где $F_{0} \simeq \Phi$, a $F_{n}$ - векторное пространство над $\Phi$ с базисом из $d^{n}$ мономов $x_{i_{1}} x_{i_{2}} \ldots x_{i_{n}}$ [2]. Обозначим $F^{(1)}=F_{1} \oplus F_{2} \oplus \cdots$. Рассмотрим ассоциативную алгебру $A=\left\langle x_{1}, \ldots, x_{d} \mid f_{1}, f_{2}, \ldots\right\rangle$ с единицей, где слева черты стоят порождающие элементы $x_{1}, \ldots, x_{d}$ алгебры $A$, а справа определяющие соотношения $f_{1}, f_{2}, \ldots$ Алгебра $A$ есть фактор-алгебра свободной алгебры $F$ по идеалу $I$, порожденному многочленами $f_{1}, f_{2}, \ldots$ [2]. Указанные соотношения таковы, что $I \leqslant F^{(1)}$ и $A^{(1)}=F^{(1)} / I$ - бесконечномерная нильалгебра. Легко проверить, что множество элементов $G=1+A^{(1)}=\left\{1+a \mid a \in A^{(1)}\right\}$ замкнуто относительно умножения и является $p$-группой. Группа $G$ называется присоединенной к алгебре $A^{(1)}$ [3]. Нильалгебра $A^{(1)}$ над полем $\Phi$ бесконечномерна, а подгруппа $P$ группы $G$, порожденная образами элементов $1+x_{1}, \ldots, 1+x_{d}$, бесконечна, поскольку линейная оболочка множества $P$ совпадает с алгеброй $A$ и $P$ содержит некоторый базис алгебры $A$. В дальнейшем элементы $f+I$ из фактор-алгебр $A=F / I, A^{(1)}$ и элементы $1+f+I \in G$ будем записывать в коротком виде $f$ и $1+f$, что не должно вызвать недоразумений.

Группа Голода $P$, как правило, не совпадает с присоединенной группой $G$ нильалгебры $A^{(1)}$. Одну из причин этого можно увидеть уже в таком простом наблюдении. Пусть для элемента $g \in A^{(1)}$ выполняется $g^{n}=0$ и $g^{n-1} \neq 0$. Тогда подалгебра в $A$, порожденная единицей и элементом $g$, содержит, по меньшей мере, $p n$ элементов, в то время как подгруппа, порожденная элементом $1+g$, состоит из $p^{k}<p n$ элементов $(1+g)^{m}=\sum_{i=0}^{i=m} C_{m}^{i} g^{i}$, где $1 \leqslant m \leqslant p^{k}$, а число $k$ определяется из неравенств

Работа поддержана Красноярским краевым фондом науки, грант 11F202C.

(С) В. А. СередА, А.И. Созутов, 2006 
$p^{k-1}<n \leqslant p^{k}$. При этом для $m<k$ имеет место равенство $(1+g)^{p^{m}}=1+g^{p^{m}} \neq 1$, а $(1+g)^{p^{k}}=1+g^{p^{k}}=1$.

Второй момент заключается в следующем. В группе $P=\left\langle 1+x_{1}, \ldots, 1+x_{d}\right\rangle$ содержатся только образы многочленов из $\Phi_{0}\left(x_{1}, \ldots, x_{d}\right)$, где $\Phi_{0}=G F(p)$ - простое подполе поля $\Phi$. Это непосредственно следует из вида порождающих группы $P$ и формулы умножения в $P:(1+f) \cdot(1+g)=1+f+g+f g$. Поэтому при изучении свойств группы Голода $P$ в смысле Шункова можно считать, что поле $\Phi$ совпадает с простым полем $G F(p)$.

Но даже в случае, когда основное поле совпадает со своим простым подполем, у авторов есть гипотеза, что подгруппа $P$ в $G$, как правило, имеет бесконечный индекс. Этим, в частности, можно объяснить долгую жизнь вопросов 8.67, 9.76 из Коуровской тетради, поскольку для всей присоединенной группы $G$ ответы на них известны давно.

Приступим непосредственно к доказательству теоремы. Итак, пусть $\Phi=G F(p)$. Предположим, что центр $Z(P)$ группы $P$ нетривиален и $1 \neq 1+f \in Z(P)$. Тогда для $1 \leqslant i \leqslant d$ выполняется

$$
1+x_{i}+f+x_{i} f=\left(1+x_{i}\right) \cdot(1+f)=(1+f) \cdot\left(1+x_{i}\right)=1+f+x_{i}+f x_{i},
$$

что влечет $f x_{i}=x_{i} f$ для всех $1 \leqslant i \leqslant d$. Отсюда выводим, что элемент $f$ перестановочен со всеми элементами алгебры $A$, а элемент $1+f$ содержится в центре $Z(G)$ всей присоединенной группы $G=1+A^{(1)}$. В частности, $Z(P)=P \cap Z(G)$.

Далее, если нильиндекс элемента $f$ равен $n$, т.е. $f^{n}=0$, а $f^{n-1} \neq 0$, то идеал $A^{(1)} f A^{(1)}=K$ алгебры $A^{(1)}$ либо совпадает с нулевым, либо нильпотентен ступени $\leqslant n$, поскольку для любых элементов $a_{1}, \ldots, a_{n}, g_{1}, \ldots, g_{n} \in A^{(1)}$ очевидно выполняется равенство

$$
\left(a_{1} f g_{1}\right) \cdots \cdot\left(a_{n} f g_{n}\right)=a_{1} g_{1} \cdots \cdot a_{n} g_{n} \cdot f^{n}=0 .
$$

Если $K=0$, то элемент $f$ принадлежит аннулятору $\operatorname{Ann}\left(A^{(2)}\right)$ подалгебры $A^{(2)}$. Поскольку аннулятор $\operatorname{Ann}\left(A^{(2)}\right)$ очевидно нильпотентен нильиндекса 3 , то в любом случае элемент $f$ принадлежит радикалу Левицкого $\mathfrak{L}\left(A^{(1))}\right.$ (локально нильпотентному радикалу алгебры $A^{(1)}[2$, с. 194].

Далее, присоединенная группа $N=1+\mathfrak{L}\left(A^{(1)}\right)$ есть нормальная подгруппа группы $G$ [3]. Очевидно, что фактор-алгебра $\bar{A}^{(1)}=A^{(1)} / \mathfrak{L}\left(A^{(1)}\right)$ порождена образами элементов $x_{1}, \ldots, x_{d}$ и является нильалгеброй. Поскольку алгебра $A^{(1)}$ не нильпотентна, то и ее фактор-алгебра $\bar{A}^{(1)}$ не нильпотентна и ввиду элементарных свойств радикала Левицкого [2] $\bar{A}^{(1)}$ не содержит ненулевых нильпотентных идеалов.

Стандартные рассуждения показывают, что присоединенная группа $\overline{1}+\bar{A}^{(1)}$ совпадает с фактор-группой $G / N$, а ее подгруппа Голода, порожденная элементами $1+\bar{x}_{1}, \ldots, \overline{1}+\bar{x}_{n}$, изоморфна фактор-группе $\bar{P}=P / W$, где $W=N \cap P$.

Если бы центр группы $\bar{P}$ был нетривиален, то как и выше, в фактор-алгебре $\bar{A}^{(1)}$ нашелся бы ненулевой нильпотентный идеал, вопреки сказанному выше. Следовательно, $Z(\bar{P})=\overline{1}$, и теорема доказана.

\section{СПИСОК ЦИТИРОВАННОЙ ЛИТЕРАТУРЫ}

[1] Коуровская тетрадъ: Нерешенные вопросы теории групп, 14-е издание, Новосибирск, 1999. [2] К. А. Жевлаков, А. М. Слинько, И. П. Шестаков, А. И. Ширшов, Кольца, близкие к ассоциативным, Наука, М., 1978. [3] А. И. Мальцев, Матем. сб., 25 (1949), $347-366$.

В. А. Середа

Поступило

Красноярский государственный аграрный университет

\section{А. И. Созутов}

Красноярская государственная архитектурно-строительная академия 